\title{
A SURVEY OF FIELD-ALIGNED MACH NUMBER AND PLASMA BETA IN THE SOLAR WIND
}

\author{
JOHAN DE KEYSER and MICHEL ROTH \\ Belgian Institute for Space Aeronomy, Brussels, Belgium \\ HANS DE STERCK and STEFAAN POEDTS \\ Katholieke Universiteit Leuven, Leuven, Belgium
}

\begin{abstract}
We have surveyed solar wind plasma beta and field-aligned Alfvénic Mach number using Ulysses and Wind data. We show the characteristic timescale and occurrence frequency of 'magnetically dominated' solar wind, whose interaction with a planetary magnetosphere may produce a bow shock with multiple shock fronts. We discuss radial, latitudinal, and solar cycle effects.
\end{abstract}

\section{Introduction}

We survey Wind and Ulysses solar wind observations to obtain the distributions of the field-aligned Alfvénic Mach number and of plasma beta. Recent magnetohydrodynamic simulations show that the interaction of 'magnetically dominated' solar wind with the Earth's magnetosphere does not lead to the well-known fasttype bow shock, but to a complex shock front with parts of different types and/or additional shocks (e.g., slow shocks) in the magnetosheath (De Sterck, 1999; De Sterck and Poedts, 1999, 2000). In this paper we (1) determine the typical duration of the 'magnetically dominated' regime, (2) establish its dependence on radial distance, heliographic latitude, and on the solar cycle, (3) identify it with solar wind structures, and (4) assess whether the theoretical complex bow shock topology actually occurs.

\section{Observations}

We use January-March 1995 Wind observations of 1 AU low-latitude solar wind (mix of slow and fast wind, typical sector structure). The 46/92 s averaged magnetometer and $90 \mathrm{~s}$ plasma data (R. Lepping and K. Ogilvie, NASA GSFC, USA) were interpolated to a common time resolution of $112.5 \mathrm{~s}$ ( $\frac{1}{32}$ hour). The Ulysses data (October 1990 to present) range from 1 to $5.4 \mathrm{AU}$ and from $-80^{\circ}$ to $+80^{\circ}$ in latitude. Intent on covering large timescales, we interpolated the $256 \mathrm{~s}$ averaged magnetic field (A. Balogh, Imperial College, London, U.K.) and $1792 \mathrm{~s}$ ion data (D. McComas, Los Alamos National Laboratory, U.S.A.) to $\frac{1}{2}$ hour resolution. 

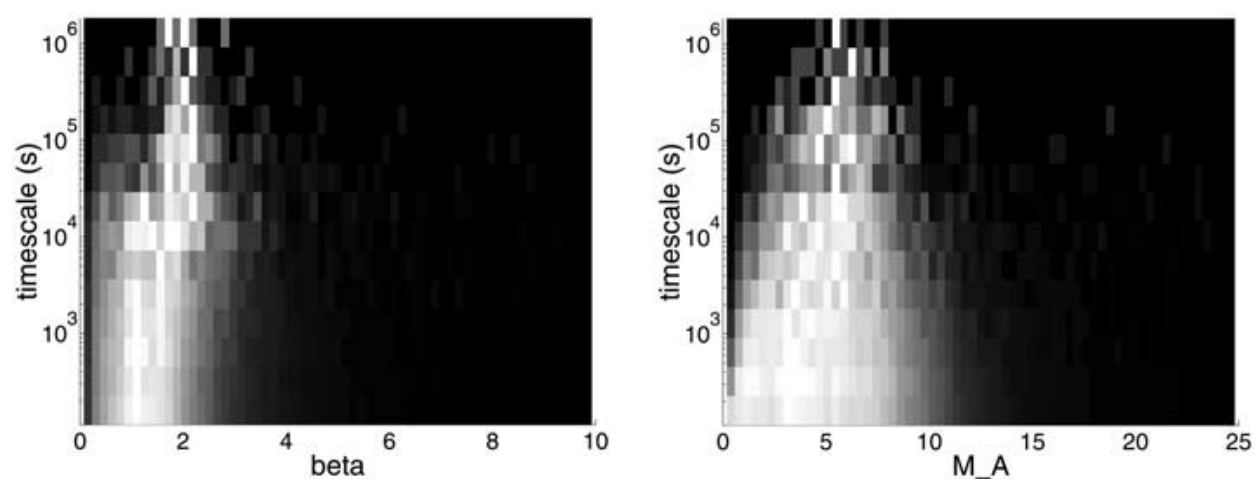

Figure 1. Multi-timescale distribution of solar wind plasma beta $\beta$ and Alfvénic Mach number $M_{\mathrm{A}}$ observed by Wind at 1 AU in January-March 1995.

Solar wind plasma beta is $\beta=P_{\mathrm{th}} / P_{\mathrm{mag}}$, with a magnetic pressure $P_{\mathrm{mag}}=$ $B^{2} / 2 \mu_{0}$. The thermal pressure $P_{\mathrm{th}}=\sum_{i} k_{B} n_{i} T_{i}$ includes contributions from all plasma populations ( $k_{B}$ is Boltzmann's constant). Because electron data were not available (data gaps, non-Maxwellian distributions, ...), we take the electron and ion pressures equal. We ignore temperature anisotropy and heavy ions. The uncertainty on $\beta$ is $\sim 30 \%$. The field-aligned Alfvénic Mach number is $M_{A}=v \cos \theta_{v B} /$ $v_{\mathrm{A}}$, where $v$ is the solar wind speed, $v_{\mathrm{A}}$ the Alfvén speed, and $\theta_{v B}$ the angle between the flow and the magnetic field. While solar wind usually is super-fast $\left(v / v_{\mathrm{A}} \gg 1\right)$, for slow wind speed, strong field, low density, and/or $\boldsymbol{B}$ nearly perpendicular to $\boldsymbol{v}, M_{\mathrm{A}}$ is small. As planetary bow shocks are curved, nature and strength of the shock change from point to point, whatever the field orientation. Where the field is perpendicular to the shock, the switch-on shock regime occurs if

$$
1<M_{\mathrm{A}}<\sqrt{\frac{\gamma(1-\beta)+1}{\gamma-1},}
$$

where $\gamma=\frac{5}{3}$; complex bow shocks can form in this regime (De Sterck and Poedts, 1999).

Figure 1 shows the distributions of $\beta$ and $M_{\mathrm{A}}$ at $1 \mathrm{AU}$ obtained by averaging the Wind data over increasingly longer time periods. Low $\beta$ and $M_{\mathrm{A}}$ conditions typically last up to $\sim 10^{4} \mathrm{~s}$, that is, a few hours at most. The switch-on regime is found about $5 \%$ of the time. Most low $M_{\mathrm{A}}$ situations occur when $\theta_{v B} \approx 90^{\circ}$, but then there exists no point on the Earth's dayside bow shock (where the shock is strongest and its structure most pronounced) where the field is perpendicular to it. Assuming the bow shock flaring angle to be about $45^{\circ}$ on the magnetospheric flanks $\left(\left|\theta_{v B}\right|<45^{\circ}\right)$, conditions for a 'magnetically dominated' dayside bow shock occur $\sim 2 \%$ of the time.

Figure 2 displays the monthly $\beta$ and $M_{\mathrm{A}}$ distributions throughout the Ulysses mission: first during the in-ecliptic trajectory (0-5.4 AU, month 0-20), then at increasingly southern latitude (above the south pole at $\sim 2 \mathrm{AU}$, month 45 ), across 

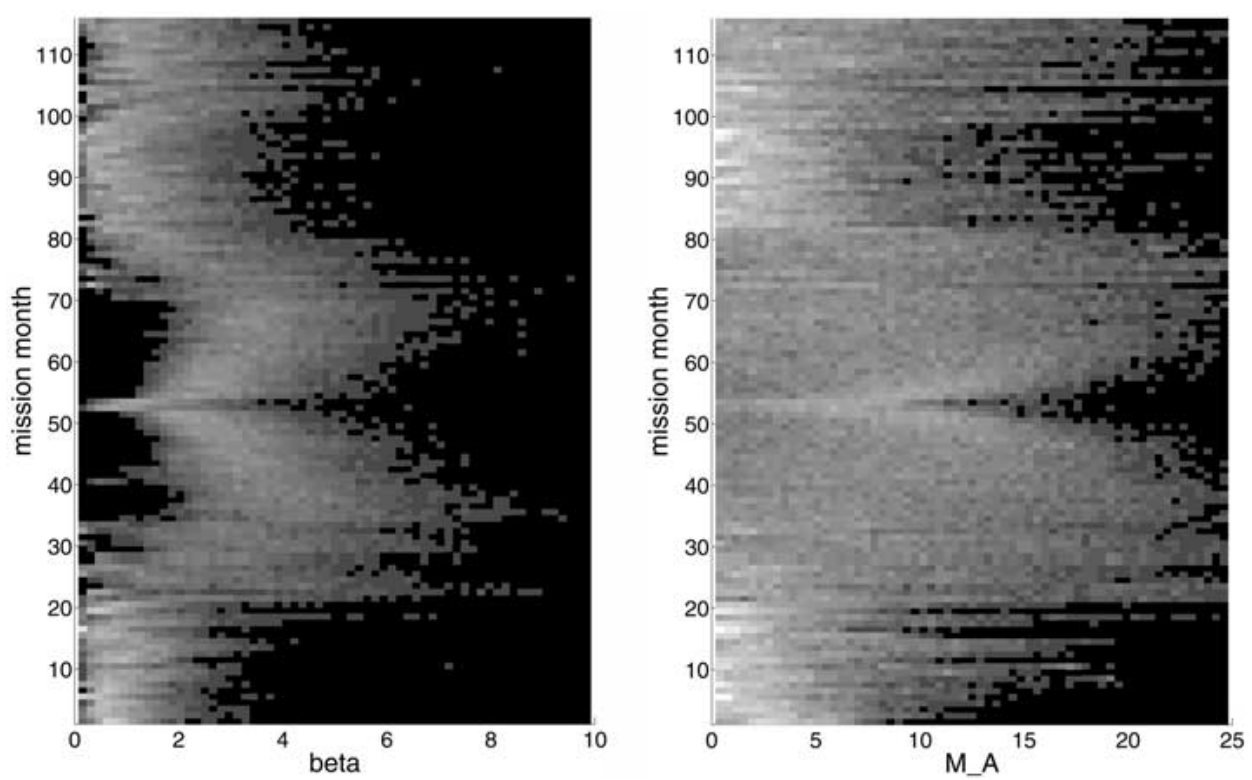

Figure 2. Distribution of plasma beta $\beta$ and Alfvénic Mach number $M_{\mathrm{A}}$ during the Ulysses mission, based on $\frac{1}{2}$ hour averaged data.

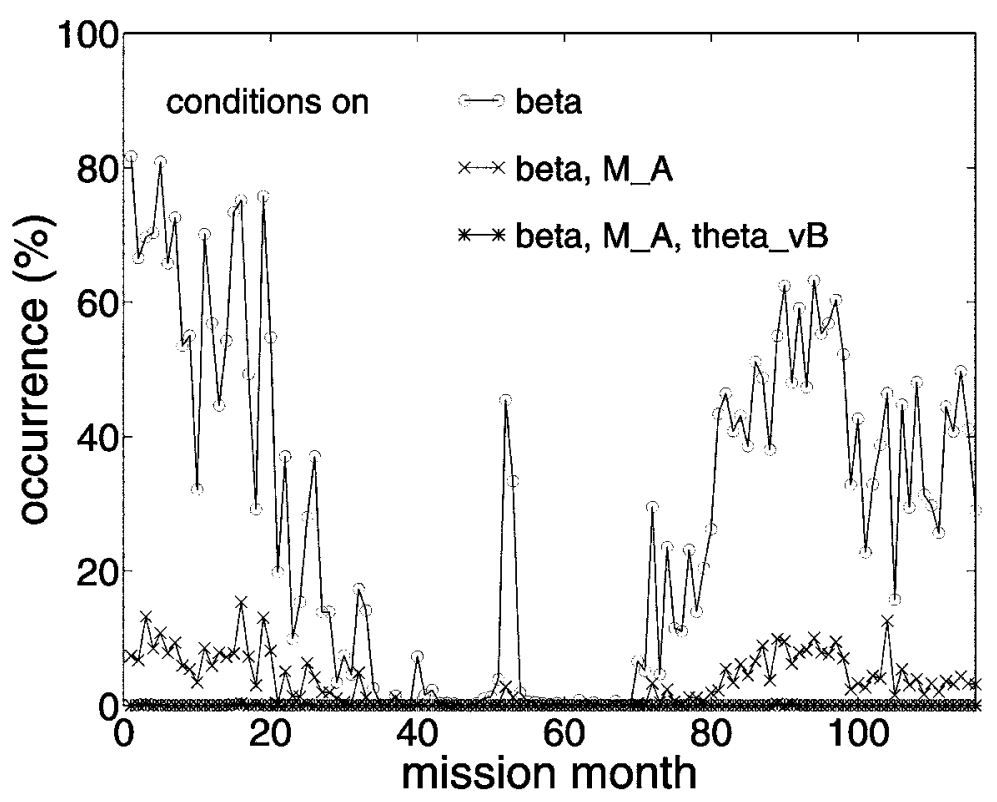

Figure 3. The fraction of time that $\beta<\sqrt{2 / \gamma}$, that $M_{\mathrm{A}}$ satisfies condition (1), and that in addition $\left|\theta_{v B}\right|<45^{\circ}$, throughout the Ulysses mission. 
the equator ( $\sim 1 \mathrm{AU}$, month 53$)$, to the north pole, and back to aphelion (5.4 $\mathrm{AU}$, around month 90). The stronger magnetic field at low latitudes leads to lower $\beta$; slow wind speed and higher $v_{\mathrm{A}}$ result in lower $M_{\mathrm{A}}$. At large distances $M_{\mathrm{A}}$ decreases as $v_{\mathrm{A}}$ increases (mainly due to lower density); the trend in $\beta$ is less clear. After month 100, solar activity becomes important: $\beta$ and $M_{\mathrm{A}}$ retain distributions similar to the quiet Sun, low-latitude ones, though somewhat broader, in spite of the increasing latitude. Figure 3 shows the occurrence of 'magnetically dominated' flow along the trajectory. As the Parker angle (typical $\theta_{v B}$ ) increases farther away from the Sun (about $80^{\circ}$ at $5 \mathrm{AU}$ ), dayside 'magnetically dominated' bow shocks become even less probable.

\section{Conclusions}

We found that near solar minimum, at $1 \mathrm{AU}$, and at low latitude, 'magnetically dominated' solar wind conditions occur up to $5 \%$ of the time, and can last a few hours. About $2 \%$ of the time the field orientation allows a complex dayside bow shock to develop (De Sterck and Poedts, 1999, 2000). If this regime does not last long enough or if it is too variable, transient slow shocks might form in the magnetosheath; evidence for these exists (Song et al., 1992). We tentatively associate 'magnetically dominated' conditions with corotating interaction regions and magnetic clouds, with their high magnetic pressure and low beta. They also have the same characteristic duration. This explains why Ulysses finds low $\beta$ and $M_{\mathrm{A}}$ only at low latitude during solar minimum but everywhere at solar maximum, when CMEs are abundant and slow/fast stream interactions exist at all latitudes.

\section{Acknowledgements}

J.D.K. and M.R. acknowledge support by ESA-PRODEX Ulysses and by the Belgian Federal Office for Scientific, Technical, and Cultural Affairs.

\section{References}

De Sterck, H.: 1999, 'Numerical Simulation and Analysis of Magnetically Dominated MHD Bow Shock Flows with Applications in Space Physics', Ph.D. thesis, Katholieke Universiteit Leuven, Leuven.

De Sterck, H. and Poedts, S.: 1999, 'Stationary Slow Shocks in the Magnetosheath for Solar Wind Conditions with $\beta<2 / \gamma$ : Three-Dimensional MHD Simulations', J. Geophys. Res. 104 (A10), $22401-22406$

De Sterck, H. and Poedts, S.: 2000, 'Intermediate Shocks in Three-Dimensional Magnetohydrodynamic Bow-Shock Flows with Multiple Interacting Shock Fronts', Phys. Rev. Lett. 84 (24), 5524 $-5527$.

Song, P., Russell, C. T., and Thomsen, M. F.: 1999, 'Slow Mode Transition in the Frontside Magnetosheath', J. Geophys. Res. 97 (A6), 8295-8305. 